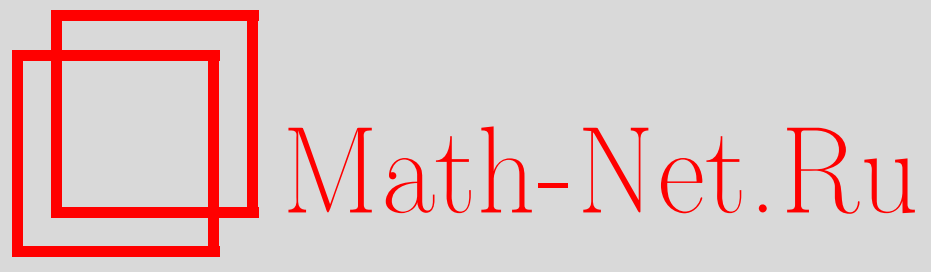

С. Е. Конштейн, А. Г. Смирнов, И. В. Тютин, Общий вид деформации суперскобки Пуассона, ТМФ, 2006, том 148, номер 2, 163-178

DOI: https://doi.org/10.4213/tmf2078

Использование Общероссийского математического портала Math-Net.Ru подразумевает, что вы прочитали и согласны с пользовательским соглашением http://www . mathnet.ru/rus/agreement

Параметры загрузки:

IP : 54.198 .187 .58

26 апреля 2023 г., 14:42:00

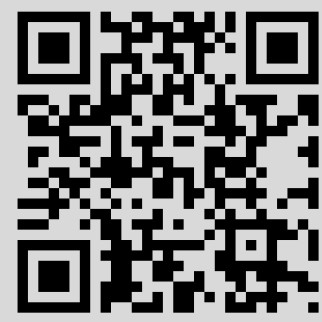




\title{
ОБЩИЙ ВИД ДЕФОРМАЦИИ СУПЕРСКОБКИ ПУАССОНА
}

\begin{abstract}
С точностью до преобразований эквивалентности найдены все непрерывные формальные деформации алгебры Пуассона, реализованной на гладких грассманозначных функциях с компактным носителем на $\mathbb{R}^{2 n}$ при $2 n \geqslant 4$. Показано, что у рассматриваемых алгебр существуют дополнительные деформации, отличные от скобки Мойала.
\end{abstract}

Ключевые слова: алгебра Грассмана, супералгебра Пуассона, центральное расширение, когомологии, деформация, *-коммутатор, квантование.

\section{1. ВВЕДЕНИЕ}

Надежда построить квантовую механику на нетривиальных многообразиях связана с геометрическим, или деформационным, квантованием [1]. С операторами ассоциируются функции на фазовом пространстве, а произведение и коммутатор операторов описываются ассоциативным *-произведением и антисимметричным *-коммутатором функций, удовлетворяющим тождеству Якоби. Эти *-произведение и *-коммутатор являются деформациями обычного произведения и обычной скобки Пуассона. В работе [2] рассмотрены супералгебра Пуассона, реализованная на полиномах, и супералгебра Пуассона с инвертированной четностью (антискобка). Чисто грассманов случай $n=0$ рассмотрен в работе [3], а чисто бозонный случай - в работе [4] при некоторых дополнительных предположениях.

В настоящей работе найден общий вид *-коммутатора супералгебры Пуассона гладких функций с компактным носителем в $\mathbb{R}^{2 n}$, принимающих значения в алгебре Грассмана, при $2 n \geqslant 4$. Предложенный анализ существенно базируется на результатах работы [5], в которой найдено второе пространство когомологий с коэффициентами в присоединенном представлении рассматриваемой супералгебры Пуассона.

\section{2. ДЕФОРМАЦИИ ТОПОЛОГИЧЕСКИХ СУПЕРАЛГЕБР ЛИ}

В этом разделе мы напомним некоторые понятия, связанные с формальными деформациями алгебр (см., например, [6]), применительно к случаю топологических

*Физический институт им. П. Н. Лебедева РАН, Москва, Россия. E-mail: konstein@lpi.ru, smirnov@lpi.ru, tyutin@lpi.ru 
супералгебр Ли. Пусть $L$ - топологическая супералгебра Ли над $\mathbb{K}(\mathbb{K}=\mathbb{R}$ или $\mathbb{C})$ с суперскобкой Ли $\{\cdot, \cdot\}, \mathbb{K}\left[\left[\hbar^{2}\right]\right]$ - кольцо формальных степеннь́х рядов по $\hbar^{2}$ над $\mathbb{K}$. Обозначим $\mathbb{K}\left[\left[\hbar^{2}\right]\right]$-модуль формальных степеннь́х рядов по $\hbar^{2}$ с коэффициентами в $L$ через $L\left[\left[\hbar^{2}\right]\right]$ и снабдим $\mathbb{K}\left[\left[\hbar^{2}\right]\right]$ и $L\left[\left[\hbar^{2}\right]\right]$ топологией прямого произведения. Градуировка $\varepsilon$ в $L$ естественно определяет градуировку в $L\left[\left[\hbar^{2}\right]\right]$ : элемент $f=f_{0}+\hbar^{2} f_{1}+\cdots$ имеет определенную четность $\varepsilon(f)$, если $\varepsilon(f)=\varepsilon\left(f_{j}\right)$ для всех $j=0,1, \ldots$ Всякое $p$-линейное раздельно-непрерывное отображение из $L^{p}$ в $L$ (в частности, скобка $\{\cdot, \cdot\})$ единственным образом продолжается вследствие $\mathbb{K}\left[\left[\hbar^{2}\right]\right]$ линейности до $p$-линейного раздельно-непрерывного отображения над $\mathbb{K}\left[\left[\hbar^{2}\right]\right]$ из $L\left[\left[\hbar^{2}\right]\right]^{p}$ в $L\left[\left[\hbar^{2}\right]\right]$. (Непрерывная) формальная деформация $L-$ это, по определению, $\mathbb{K}\left[\left[\hbar^{2}\right]\right]$-билинейная непрерывная по каждому аргументу в отдельности суперскобка Ли $C(\cdot, \cdot)$ на $L\left[\left[\hbar^{2}\right]\right]$ такая, что $C(f, g)=\{f, g\} \bmod \hbar^{2}$ для любых $f, g \in L\left[\left[\hbar^{2}\right]\right]$. Очевидно, что всякая формальная деформация $C$ представима в виде

$$
C(f, g)=\{f, g\}+\hbar^{2} C_{1}(f, g)+\hbar^{4} C_{2}(f, g)+\cdots, \quad f, g \in L,
$$

где $C_{j}$ - раздельно-непрерывные суперантисимметричные билинейные отображения из $L \times L$ в $L$ (2-коцепи с коэффициентами в присоединенном представлении $L)$. Формальные деформации $C^{1}$ и $C^{2}$ называются эквивалентными, если существует непрерывный $\mathbb{K}\left[\left[\hbar^{2}\right]\right]$-линейный оператор $T=\mathrm{id}+\hbar^{2} T_{1}+\hbar^{4} T_{2}+\cdots$ из $L\left[\left[\hbar^{2}\right]\right]$ в $L\left[\left[\hbar^{2}\right]\right]$ такой, что $T C^{1}(f, g)=C^{2}(T f, T g), f, g \in L\left[\left[\hbar^{2}\right]\right]$.

Задача поиска формальных деформаций $L$ тесно связана с задачей вычисления когомологий Шевалле-Эйленберга супералгебры $L$ с коэффициентами в присоединенном представлении $L$. Пусть $\mathcal{C}_{p}(L)$ обозначает пространство $p$-линейных суперантисимметричных раздельно-непрерывных отображений из $L^{p}$ в $L$ (пространство $p$-коцепей с коэффициентами в присоединенном представлении $L$ ). Пространство $\mathcal{C}_{p}(L)$ имеет естественную $\mathbb{Z}_{2}$-градуировку: по определению $M_{p} \in \mathcal{C}_{p}(L)$ имеет определенную четность $\epsilon\left(M_{p}\right)$, если соотношение

$$
\varepsilon\left(M_{p}\left(f_{1}, \ldots, f_{p}\right)\right)=\varepsilon\left(M_{p}\right)+\varepsilon\left(f_{1}\right)+\cdots+\varepsilon\left(f_{p}\right)
$$

справедливо при любых $f_{j} \in L$ с определенными четностями $\epsilon\left(f_{j}\right)$.

В настоящей работе мы рассматриваем только четные деформации четной суперскобки Ли, поэтому все $C_{j}$ в разложении (1) должны быть четными 2-коцепями.

Дифференциал $d_{p}^{\text {ad }}$ определяется как такой линейный оператор из $\mathcal{C}_{p}(L){ }_{\text {в }} \mathcal{C}_{p+1}(L)$, что

$$
\begin{aligned}
& d_{p}^{\mathrm{ad}} M_{p}\left(f_{1}, \ldots, f_{p+1}\right)= \\
& =-\sum_{j=1}^{p+1}(-1)^{j+\varepsilon\left(f_{j}\right)|\varepsilon(f)|_{1, j-1}+\varepsilon\left(f_{j}\right) \varepsilon_{M_{p}}}\left\{f_{j}, M_{p}\left(f_{1}, \ldots, \breve{f}_{j}, \ldots, f_{p+1}\right)\right\}- \\
& -\sum_{i<j}(-1)^{j+\varepsilon\left(f_{j}\right)|\varepsilon(f)|_{i+1, j-1}} M_{p}\left(f_{1}, \ldots, f_{i-1},\left\{f_{i}, f_{j}\right\}, f_{i+1}, \ldots, \breve{f}_{j}, \ldots, f_{p+1}\right)
\end{aligned}
$$


для любых $M_{p} \in \mathcal{C}_{p}(L)$ и $f_{1}, \ldots, f_{p+1} \in L$, имеющих определенные четности. Здесь использовано обозначение

$$
|\varepsilon(f)|_{i, j}=\sum_{l=i}^{j} \varepsilon\left(f_{l}\right)
$$

и знак ` обозначает отсутствие аргумента, над которым он поставлен. Выписывая тождество Якоби для деформации $C$ вида (1),

$$
(-1)^{\varepsilon(f) \varepsilon(h)} C(f, C(g, h))+\operatorname{cycle}(f, g, h)=0,
$$

и рассматривая члены порядка $\hbar^{2}$, мы находим, что

$$
d_{2}^{\mathrm{ad}} C_{1}=0
$$

Таким образом, первый порядок деформации супералгебры $L$ является 2 -коциклом дифференциала $d^{\mathrm{ad}}$.

Легко также видеть, что первый порядок разности эквивалентных деформаций является кограницей.

\section{3. СУПЕРАЛГЕБРА ПУАССОНА}

Обозначим через $\mathcal{D}\left(\mathbb{R}^{k}\right)$ пространство гладких $\mathbb{K}$-значных функций на $\mathbb{R}^{k}$ с компактным носителем, снабженное своей стандартной топологией: последовательность $\varphi_{k} \in \mathcal{D}\left(\mathbb{R}^{k}\right)$ сходится к $\varphi \in \mathcal{D}\left(\mathbb{R}^{k}\right)$, если $\partial^{\lambda} \varphi_{k}$ сходится равномерно к $\partial^{\lambda} \varphi$ для любого мультииндекса $\lambda$ и носители всех $\varphi_{k}$ содержатся в некотором компактном множестве. Положим

$$
\mathbf{D}_{n_{+}}^{n_{-}}=\mathcal{D}\left(\mathbb{R}^{n_{+}}\right) \otimes \mathbb{G}^{n_{-}}, \quad \mathbf{E}_{n_{+}}^{n_{-}}=C^{\infty}\left(\mathbb{R}^{n_{+}}\right) \otimes \mathbb{G}^{n_{-}},
$$

где $\mathbb{G}^{n_{-}}$- алгебра Грассмана с $n_{-}$образующими. Образующие алгебры Грассмана (соответственно координаты пространства $\mathbb{R}^{n_{+}}$) мы обозначаем через $\xi^{\alpha}, \alpha=$ $1, \ldots, n_{-}\left(\right.$соответственно $\left.x^{i}, i=1, \ldots, n_{+}\right)$. Мы будем также использовать коллективные переменные $z^{A}$, которые равны $x^{A}$ при $A=1, \ldots, n_{+}$и $\xi^{A-n_{+}}$при $A=n_{+}+1, \ldots, n_{+}+n_{-}$. Пространства $\mathbf{D}_{n_{+}}^{n_{-}}$и $\mathbf{E}_{n_{+}}^{n_{-}}$обладают естественной градуировкой, определяемой градуировкой алгебры Грассмана. Четность элемента $f$ этих пространств обозначается через $\varepsilon(f)$. Положим также $\varepsilon_{A}=0$ для $A=1, \ldots, n_{+}$ и $\varepsilon_{A}=1$ для $A=n_{+}+1, \ldots, n_{+}+n_{-}$.

Пусть $\partial_{A} \equiv \partial / \partial z^{A}$ и $\overleftarrow{\partial}_{A} \equiv \overleftarrow{\partial} / \partial z^{A}-$ операторы левого и правого дифференцирований. Скобка Пуассона определяется соотношением

$$
\{f, g\}=f(z) \overleftarrow{\partial}_{A} \omega^{A B} \partial_{B} g(z)=-(-1)^{\varepsilon(f) \varepsilon(g)}\{g, f\}
$$

где симплектическая метрика $\omega^{A B}=-(-1)^{\varepsilon_{A} \varepsilon_{B}} \omega^{B A}$ является постоянной невырожденной матрицей. Для определенности выберем ее в виде

$$
\omega^{A B}=\left(\begin{array}{cc}
\omega^{i j} & 0 \\
0 & \lambda_{\alpha} \delta^{\alpha \beta}
\end{array}\right), \quad \lambda_{\alpha}= \pm 1, \quad i, j=1, \ldots, n_{+}, \quad \alpha, \beta=1, \ldots, n_{-},
$$


где $\omega^{i j}$ - каноническая симплектическая форма (если $\mathbb{K}=\mathbb{C}$, то можно выбрать $\left.\lambda_{\alpha}=1\right)$. Невырожденность матрицы $\omega^{A B}$ означает, в частности, что $n_{+}$четно. Скобка Пуассона удовлетворяет тождеству Якоби

$$
(-1)^{\varepsilon(f) \varepsilon(h)}\{f,\{g, h\}\}+\operatorname{cycle}(f, g, h)=0, \quad f, g, h \in \mathbf{E}_{n_{+}}^{n_{-}} .
$$

Супералгеброй Пуассона $\mathcal{P}$ мы называем пространство $\mathbf{D}_{n_{+}}^{n_{-}}$со скобкой Пуассона (5) на нем. Соотношения (5) и (6) показывают, что эта скобка действительно определяет структуру супералгебры Ли на $\mathbf{D}_{n_{+}}^{n_{-}}$.

Интеграл на $\mathbf{D}_{n_{+}}^{n_{-}}$определяется соотношением

$$
\bar{f} \stackrel{\text { def }}{=} \int d z f(z)=\int_{\mathbb{R}^{n_{+}}} d x \int d \xi f(z),
$$

где интеграл по алгебре Грассмана нормирован условием $\int d \xi \xi^{1} \ldots \xi^{n_{-}}=1$.

\section{4. ФОРМУЛИРОВКА РЕЗУЛЬТАТОВ}

Ниже мы будем использовать обозначения

$$
\begin{aligned}
& m_{2 \mid 1}(f, g) \stackrel{\text { def }}{=} f(z)\left(\overleftarrow{\partial}_{A} \omega^{A B} \partial_{B}\right)^{3} g(z), \\
& m_{2 \mid 3}(f, g) \stackrel{\text { def }}{=} \bar{g} \mathcal{E}_{z} f(z)-\bar{f} \mathcal{E}_{z} g(z), \quad \mathcal{E}_{z}=1-\frac{1}{2} z^{A} \partial_{A}, \\
& m_{2 \mid \zeta}(f, g) \stackrel{\text { def }}{=}\{\zeta(z), f(z)\} \bar{g}-(-1)^{\varepsilon(f) \varepsilon(g)}\{\zeta(z), g(z)\} \bar{f}, \quad \zeta \in \mathbf{E}_{n_{+}}^{n_{-}},
\end{aligned}
$$

и, кроме того, обозначение

$$
J(P, Q \mid f, g, h) \stackrel{\text { def }}{=}(-1)^{\varepsilon(f) \varepsilon(h)}[P(f, Q(g, h))+Q(f, P(g, h))]+\operatorname{cycle}(f, g, h)
$$

для якобиатора 2-форм. Если $P=m_{2 \mid i}$, а $Q=m_{2 \mid j}$, то мы будем писать $J_{i, j}(f, g, h)$ вместо $J(P, Q \mid f, g, h)$.

Формы $m_{2 \mid 1}(f, g), m_{2 \mid 3}(f, g)$, и $m_{2 \mid \zeta}(f, g)$ и их якобиаторы удовлетворяют соотношениям

$$
\begin{gathered}
\overline{m_{2 \mid 1}(f, g)}=\overline{m_{2 \mid 3}(f, g)}=\overline{m_{2 \mid \zeta}(f, g)}=0, \\
J_{\zeta, \zeta}(f, g, h)=J_{\zeta, 3}(f, g, h)=J_{3,3}(f, g, h)=0, \\
J_{1,3}(f, g, h)=-2(-1)^{\varepsilon(f) \varepsilon(h)} m_{2 \mid 1}(f, g) \bar{h}+\operatorname{cycle}(f, g, h) ; \\
J_{1, \zeta}(f, g, h)=(-1)^{\varepsilon(f) \varepsilon(h)} d_{2}^{\text {ad }} j_{1, \zeta}(f, g, h),
\end{gathered}
$$

где

$$
j_{1, \zeta}(f, g)=m_{2 \mid 1}(f, \zeta) \bar{g}-(-1)^{\varepsilon(f) \varepsilon(g)} m_{2 \mid 1}(g, \zeta) \bar{f} .
$$

При выводе этих формул мы использовали тождество

$$
\begin{aligned}
\mathcal{E}_{z}\left[f(z)\left(\overleftarrow{\partial}_{A} \omega^{A B} \partial_{B}\right)^{p} g(z)\right]=\left(\mathcal{E}_{z} f(z)\right)\left(\overleftarrow{\partial}_{A} \omega^{A B} \partial_{B}\right)^{p} g(z)+ \\
\quad+f(z)\left(\overleftarrow{\partial}_{A} \omega^{A B} \partial_{B}\right)^{p} \mathcal{E}_{z} g(z)+(p-1) f(z)\left(\overleftarrow{\partial}_{A} \omega^{A B} \partial_{B}\right)^{p} g(z)
\end{aligned}
$$


Суперскобка Мойала

$$
\mathcal{M}_{\kappa}(f, g)=\frac{1}{\hbar \kappa} f(z) \operatorname{sh}\left(\hbar \kappa \overleftarrow{\partial}_{A} \omega^{A B} \partial_{B}\right) g(z)
$$

суперантисимметрична, удовлетворяет тождеству Якоби при произвольном $\kappa \in$ $\mathbb{K}\left[\left[\hbar^{2}\right]\right]^{1)}$ и, следовательно, является деформацией исходной алгебры Пуассона. Для $\zeta \in \mathbf{E}_{n_{+}}^{n_{-}}\left[\left[\hbar^{2}\right]\right], \kappa, c \in \mathbb{K}\left[\left[\hbar^{2}\right]\right]$, положим

$$
\begin{aligned}
\mathcal{N}_{\kappa, \zeta}(f, g) & =\mathcal{M}_{\kappa}\left(f-\hbar^{2} \zeta \bar{f}, g-\hbar^{2} \zeta \bar{g}\right), \\
\mathcal{N}_{\kappa, \zeta, c}(f, g) & =\mathcal{M}_{\kappa}\left(f-\hbar^{2} \zeta \bar{f}, g-\hbar^{2} \zeta \bar{g}\right)+c \bar{f} \bar{g}
\end{aligned}
$$

Обозначим через $\mathbf{Z}_{n_{+}}^{n_{-}}$подалгебру $\mathbf{Z}_{n_{+}}^{n_{-}}=\mathbf{D}_{n_{+}}^{n_{-}} \oplus \mathcal{C}_{\mathbf{E}_{n_{+}}^{n_{-}}}\left(\mathbf{D}_{n_{+}}^{n_{-}}\right) \in \mathbf{E}_{n_{+}}^{n_{-}}$, где $\mathcal{C}_{\mathbf{E}_{n_{+}}^{n_{-}}}\left(\mathbf{D}_{n_{+}}^{n_{-}}\right)-$ централизатор $\mathbf{D}_{n_{+}}^{n_{-}}$в $\mathbf{E}_{n_{+}}^{n_{-}}$. Очевидно, что $\mathcal{C}_{\mathbf{E}_{n_{+}}^{n_{-}}}\left(\mathbf{D}_{n_{+}}^{n_{-}}\right)$изоморфно $\mathbb{K}$.

Теперь мы можем сформулировать основной результат настоящей работы.

Теорема 1. 1. Пусть $n_{+} \geqslant 4, n_{-}=2 k$. Тогда всякая непрерывная формальная дебормащия супералгебры Пуассона $\mathcal{P}$ эквивалентна либо суперскобке $\mathcal{N}_{\kappa, \zeta}(f, g)$ при некоторой четной бункиии $\zeta \in \mathbf{E}_{n_{+}}^{n_{-}}\left[\left[\hbar^{2}\right]\right]$ u $\kappa \in \mathbb{K}\left[\left[\hbar^{2}\right]\right]$, либо суперскобке

$$
C_{\zeta, c}(f, g)=\{f(z), g(z)\}+m_{2 \mid \zeta}(f, g)+c m_{2 \mid 3}(f, g)
$$

при некоторой четной функиии $\zeta \in \mathbf{E}_{n_{+}}^{n_{-}}\left[\left[\hbar^{2}\right]\right]$ u $c \in \mathbb{K}\left[\left[\hbar^{2}\right]\right]$.

Суперскобки $\mathcal{N}_{\kappa_{1}, \zeta_{1}}(f, g)$ и $\mathcal{N}_{\kappa_{2}, \zeta_{2}}(f, g)$ эквивалентны, если $\kappa_{1}=\kappa_{2} u \zeta_{1}-\zeta_{2} \in \mathbf{Z}_{n_{+}}^{n_{-}}$.

Суперскобки $C_{\zeta_{1}, c_{1}}(f, g)$ и $C_{\zeta_{2}, c_{2}}(f, g)$ эквивалентны, если $c_{1}=c_{2} u \zeta_{1}-\zeta_{2} \in \mathbf{Z}_{n_{+}}^{n_{-}}$.

2. Пусть $n_{+} \geqslant 4, n_{-}=2 k+1$. Тогда всякая непрерьвная формальная деформация супералгебры Пуассона $\mathcal{P}$ эквивалентна суперскобке $\mathcal{N}_{\kappa, \zeta, c}(f, g)$ при некоторьх $c, \kappa \in \mathbb{K}\left[\left[\hbar^{2}\right]\right]$ и нечетной функиии $\zeta \in \mathbf{E}_{n_{+}}^{n_{-}}\left[\left[\hbar^{2}\right]\right]$ таких, что $\hbar^{4} \mathcal{M}_{\kappa}(\zeta, \zeta)+c \in$ $\mathbf{D}_{n_{+}}^{n_{-}}\left[\left[\hbar^{2}\right]\right]$.

Суперскобки $\mathcal{N}_{\kappa_{1}, \zeta_{1}, c_{1}}(f, g)$ и $\mathcal{N}_{\kappa_{2}, \zeta_{2}, c_{2}}(f, g)$ эквивалентны, если $\kappa_{1}=\kappa_{2}, c_{1}=c_{2} u$ $\zeta_{1}-\zeta_{2} \in \mathbf{Z}_{n_{+}}^{n_{-}}$.

Оставшаяся часть работы представляет собой доказательство этой теоремы.

\section{5. СЛУЧАЙ $n_{-}=2 k$}

Прежде всего заметим, что суперскобки $\mathcal{N}_{\kappa, \zeta}(f, g)$ и $C_{\zeta, c}(f, g)$ удовлетворяют тождеству Якоби. Для доказательства достаточно заметить, что

$$
\int d z f(z)\left(\overleftarrow{\partial}_{A} \omega^{A B} \partial_{B}\right)^{p} \zeta(z)=0
$$

для всех $p \geqslant 1, f \in \mathbf{D}_{n_{+}}^{n_{-}}, \zeta \in \mathbf{E}_{n_{+}}^{n_{-}}$.

1)Этот факт хорошо известен для бозонного случая и может быть легко проверен для суперслучая. 
Из теоремы 3 работы [5] следует, что общее решение уравнения (1) для первого порядка деформации задается выражением²)

$$
\begin{gathered}
C_{1}(f, g)=\frac{1}{6} \kappa_{1}^{2} m_{2 \mid 1}(f, g)+m_{2 \mid \zeta_{1}}(f, g)+c_{31} m_{2 \mid 3}(f, g)+d_{1}^{\mathrm{ad}} t_{D 1}(f, g)+ \\
+c_{41} \delta_{2 k-n_{+}, 4} m_{2 \mid 4}(f, g), \\
\varepsilon\left(\kappa_{1}\right)=\varepsilon\left(c_{31}\right)=\varepsilon\left(\zeta_{1}(z)\right)=0,
\end{gathered}
$$

где

$$
m_{2 \mid 4}(f, g)=\int d u\left(\mathcal{E}_{u} f(u) g(u)-(-1)^{\varepsilon(f) \varepsilon(g)} \mathcal{E}_{u} g(u) f(u)\right), \quad t_{D 1}(f) \in \mathbf{D}_{n_{+}}^{n_{-}} .
$$

Условие $C(f, g) \in \mathbf{D}_{n_{+}}^{n_{-}}$дает, что $c_{41}=0$.

Выполнив преобразование эквивалентности $C(f, g) \rightarrow C_{T}(f, g)$ с $T(f)=f(z)-$ $\hbar^{2} t_{D 1}(f)+O\left(\hbar^{4}\right)$, мы можем переписать $C(f, g)$ в виде

$$
C(f, g)=\mathcal{N}_{\kappa_{1}, \zeta_{1}}(f, g)+c_{31} \hbar^{2} m_{2 \mid 3}(f, g)+\hbar^{4} C_{2}(f, g)+O\left(\hbar^{6}\right) .
$$

Из тождества Якоби (3) для $C(f, g, h)$ получаем

$$
d_{2}^{\mathrm{ad}} C_{2}(f, g, h)=-\frac{c_{31} \kappa_{1}^{2}}{3}(-1)^{\varepsilon(f) \varepsilon(h)} J_{1,3}(f, g, h)-\frac{\kappa_{1}^{2}}{6} d_{2}^{\mathrm{ad}} j_{1, \zeta_{1}}(f, g, h) .
$$

Пусть

$$
[z \cup \operatorname{supp}(f) \cup \operatorname{supp}(g)] \cap \operatorname{supp}(h)=\varnothing .
$$

Из уравнения (12) следует, что

$$
\begin{gathered}
d_{1}^{\text {ad }} \widehat{C}_{2}(f, g, h)=\frac{c_{31} \kappa_{1}^{2}}{3}(-1)^{\varepsilon(f) \varepsilon(h)} m_{2 \mid 1}(f, g) \bar{h}-\frac{\kappa_{1}^{2}}{6} d_{1}^{\text {ad }} j_{\zeta_{1}}(f, g) \bar{h}, \\
j_{\zeta}(f, g) \stackrel{\text { def }}{=} m_{2 \mid 1}(f, \zeta),
\end{gathered}
$$

где дифференциал $d_{1}^{\mathrm{ad}}$ в левой части уравнения (13) действует только на первый аргумент функционала $\widehat{C}_{2}(f, h)$. Здесь мы, как и в работе [5], используем обозначение ^ для ограничения формы на указанную область.

Как показано в п. 4.3 работы [5], уравнение (13) имеет решение только тогда, когда

$$
c_{31} \kappa_{1}=0
$$

Рассмотрим по отдельности следующие три случая.

1. $\kappa_{1}=0, c_{31} \neq 0$. В этом случае уравнение (12) принимает вид

$$
d_{2}^{\mathrm{ad}} C_{2}(f, g, h)=0,
$$

2) При выводе выражения (11) в работе [5] было использовано утверждение о том, что если форма $M_{2}(z \mid f, g)$, рассматриваемая как функция аргумента $z$, является гладкой и имеет место представление $M_{2}(z \mid f, g)=d_{1}^{\text {ad }} M_{1}(z \mid f, g)$, то форма $M_{1}(z \mid f)$ также является гладкой по аргументу $z$. Точная формулировка и доказательство этого утверждения приведены в приложении. 
и его общее решение в $\mathbf{D}_{n_{+}}^{n_{-}}$

$$
C_{2}(f, g)=\kappa_{2} m_{2 \mid 1}(f, g)+m_{2 \mid \zeta_{2}}(f, g)+c_{32} m_{2 \mid 3}(f, g)+d_{1}^{\mathrm{ad}} t_{D 2}(f, g) .
$$

Выполняя преобразование эквивалентности $C(f, g) \rightarrow C_{T}(f, g)$ с $T(f)=f(z)-$ $\hbar^{4} t_{D 2}(f)+O\left(\hbar^{6}\right)$, перепишем $C(f, g)$ в виде

$$
\begin{gathered}
C(f, g)=\{f(z), g(z)\}+\hbar^{4} \kappa_{2} m_{2 \mid 1}(f, g)+m_{2 \mid \zeta_{[2]}}(f, g)+ \\
+c_{3[2]} m_{2 \mid 3}(f, g)+\hbar^{6} C_{3}(f, g)+O\left(\hbar^{8}\right), \\
\zeta_{[n]}(z)=\sum_{k=1}^{n} \hbar^{2 k} \zeta_{k}(z), \quad c_{3[n]}=\sum_{k=1}^{n} \hbar^{2 k} c_{3 k} .
\end{gathered}
$$

Тождество Якоби (3) для $C(f, g, h)$ дает

$$
d_{2}^{\mathrm{ad}} C_{3}(f, g, h)=-c_{31} \kappa_{2}(-1)^{\varepsilon(f) \varepsilon(h)} J_{1,3}(f, g, h)-d_{2}^{\mathrm{ad}} J_{1, \zeta_{1}}(f, g, h) .
$$

Теперь мы можем заключить, что уравнение (14) имеет решение только тогда, когда $c_{31} \kappa_{2}=0$. Из этого следует, что $\kappa_{2}=0$. Продолжая тем же образом, мы находим, что с точностью до преобразования эквивалентности общий вид функционала $C(f, g)$, удовлетворяющего тождеству Якоби (3) (общий вид *-коммутатора), задан выражением

$$
C(f, g)=\{f(z), g(z)\}+m_{2 \mid \zeta_{[\infty]}}(f, g)+c_{3[\infty]} m_{2 \mid 3}(f, g) .
$$

Соотношения (9) позволяют провести прямую проверку того, что форма (15) удовлетворяет тождеству Якоби (3).

2. $\kappa_{1} \neq 0, c_{31}=0$. В этом случае уравнение (12) принимает вид

$$
d_{2}^{\text {ad }} C_{2}(f, g, h)=-\frac{\kappa_{1}^{2}}{6} d_{2}^{\text {ad }} j_{1, \zeta_{1}}(f, g, h)
$$

и имеет следующее общее решение:

$$
C_{2}(f, g)=-\frac{\kappa_{1}^{2}}{6} j_{1, \zeta_{1}}(f, g)+\frac{\kappa_{1} \kappa_{2}}{3} m_{2 \mid 1}(f, g)+m_{2 \mid \zeta_{2}}(f, g)+c_{32} m_{2 \mid 3}(f, g)+d_{1}^{\text {ad }} t_{D 2}(f, g) .
$$

Выполнив преобразование эквивалентности $C(f, g) \rightarrow C_{T}(f, g)$ с $T(f)=f(z)-$ $\hbar^{4} t_{D 2}(f)+O\left(\hbar^{6}\right)$, мы можем представить $C(f, g)$ в виде ${ }^{3)}$

$$
C(f, g)=\mathcal{N}_{\kappa_{[2]}, \zeta_{[2]}}(f, g)+c_{32} \hbar^{4} m_{2 \mid 3}(f, g)+\hbar^{6} C_{3}(f, g)+O\left(\hbar^{8}\right),
$$

где

$$
\kappa_{[n]}=\sum_{k=1}^{n} \hbar^{2(k-1)} \kappa_{k} .
$$

3) При выводе этого соотношения мы использовали равенство

$$
\zeta(z)\left(\overleftarrow{\partial}_{A} \omega^{A B} \partial_{B}\right)^{p} \zeta(z)=0
$$

справедливое при $\varepsilon(\zeta)=0, p=2 k+1$. 
Тождество Якоби (3) для $C(f, g, h)$ дает

$$
d_{2}^{\mathrm{ad}} C_{3}(f, g, h)=-\frac{c_{32} \kappa_{1}^{2}}{6}(-1)^{\varepsilon(f) \varepsilon(h)} J_{1,3}(f, g, h) .
$$

Это уравнение имеет решение только при $c_{32} \kappa_{1}=0$, откуда следует, что $c_{32}=0$.

Действуя тем же образом, мы находим, что с точностью до преобразования эквивалентности общий вид функционала $C(f, g)$, удовлетворяющего тождеству Якоби (3) (общий вид *-коммутатора), задан выражением

$$
C(f, g)=\mathcal{N}_{\kappa_{[\infty]}, \zeta_{[\infty]}}(f, g), \quad \zeta_{[\infty]}(z) \in \mathbf{E}_{n_{+}}^{n_{-}} / \mathbf{Z}_{n_{+}}^{n_{-}}, \quad \varepsilon\left(\zeta_{[\infty]}\right)=0 .
$$

3. $\kappa_{1}=c_{31}=0$. В этом случае уравнение (12) принимает вид

$$
d_{2}^{\mathrm{ad}} C_{2}(f, g, h)=0
$$

и имеет общее решение

$$
C_{2}(f, g)=\frac{\kappa_{2}^{2}}{6} m_{2 \mid 1}(f, g)+m_{2 \mid \zeta_{2}}(f, g)+c_{32} m_{2 \mid 3}(f, g)+d_{1}^{\mathrm{ad}} t_{D 2}(f, g) .
$$

Выполнив преобразование эквивалентности $C(f, g) \rightarrow C_{T}(f, g)$ с $T(f)=f(z)-$ $\hbar^{4} t_{D 2}(f)+O\left(\hbar^{6}\right)$, перепишем $C(f, g)$ в виде

$$
C(f, g)=\mathcal{N}_{\hbar \kappa_{2}, \zeta_{[2]}}(f, g)+c_{32} \hbar^{4} m_{2 \mid 3}(f, g)+\hbar^{6} C_{3}(f, g)+O\left(\hbar^{8}\right) .
$$

Тождество Якоби (3) для $C(f, g, h)$ дает

$$
\begin{gathered}
d_{2}^{\text {ad }} C_{3}(f, g, h)=0, \\
C_{3}(f, g)=\frac{\kappa_{2} \kappa_{3}}{3} m_{2 \mid 1}(f, g)+m_{2 \mid \zeta_{3}}(f, g)+c_{33} m_{2 \mid 3}(f, g)+d_{1}^{\text {ad }} t_{D 3}(f, g) .
\end{gathered}
$$

Выполнив преобразование эквивалентности $C(f, g) \rightarrow C_{T}(f, g)$ с $T(f)=f(z)-$ $\hbar^{6} t_{D 3}(f)+O\left(\hbar^{8}\right)$, перепишем $C(f, g)$ в виде

$$
C(f, g)=\mathcal{N}_{\hbar \kappa_{[3]}, \zeta_{[3]}}(f, g)+c_{3[3]} m_{2 \mid 3}(f, g)+\hbar^{8} C_{4}(f, g)+O\left(\hbar^{10}\right) .
$$

Тождество Якоби (3) для $C(f, g)$ дает

$$
\begin{gathered}
d_{2}^{\mathrm{ad}} C_{4}(f, g, h)=-\frac{\kappa_{2}^{2} c_{32}}{6}(-1)^{\varepsilon(f) \varepsilon(h)} J_{1,3}(f, g, h) \Longrightarrow \\
\Longrightarrow \quad \kappa_{2} c_{32}=0
\end{gathered}
$$

и т.д.

\section{6. СЛУЧАЙ $n_{-}=2 k+1$}

В этом случае общее решение уравнения (1) для первого порядка деформации задано выражением

$$
\begin{gathered}
C_{1}(f, g)=\frac{1}{6} \kappa_{1}^{2} m_{2 \mid 1}(f, g)+m_{2 \mid \zeta_{C 1}}(f, g)+d_{1}^{\mathrm{ad}} t_{D 1}(f, g), \\
\varepsilon\left(\kappa_{1}\right)=0, \quad \varepsilon\left(\zeta_{C 1}(z)\right)=1 .
\end{gathered}
$$


Заметим, что, в противоположность $(11)$, член $m_{2 \mid 3}(f, g)$ не входит в первый порядок деформации, так как из-за нечетности $n_{-}$он не имеет необходимой четности.

Рассмотрим форму

$$
\begin{aligned}
\mathcal{N}_{\kappa, \zeta, c_{2}}(f, g) & =\mathcal{M}_{\kappa}\left(f-\hbar^{2} \zeta_{C} \bar{f}, g-\hbar^{2} \zeta_{C} \bar{g}\right)+c_{2} \bar{f} \bar{g}= \\
& =\mathcal{M}_{\kappa}(f, g)+\mathcal{M}_{\kappa}\left(f, \zeta_{C}\right) \bar{g}-(-1)^{\varepsilon(f) \varepsilon(g)} \mathcal{M}_{\kappa}\left(g, \zeta_{C}\right) \bar{f}+\mathcal{L}_{\kappa, \zeta, c_{2}}(z) \bar{f} \bar{g},
\end{aligned}
$$

где

$$
\mathcal{L}_{\kappa, \zeta, c_{2}}(z)=\mathcal{M}_{\kappa}\left(\zeta_{C}, \zeta_{C}\right)+c_{2}, \quad \varepsilon\left(\zeta_{C}(z)\right)=1 .
$$

Мы предполагаем, что $\zeta_{C}(z) \in \mathbf{E}_{n_{+}}^{n_{-}}$, а $\mathcal{L}_{\kappa, \zeta, c_{2}}(z) \in \mathbf{D}_{n_{+}}^{n_{-}}$.

ПРЕДЛОЖЕНИЕ. Форма $\mathcal{N}_{\kappa, \zeta, c_{2}}(f, g)$ имеет следующие свойства:

1) $\mathcal{N}_{\kappa, \zeta, c_{2}}(f, g) \in \mathbf{D}_{n_{+}}^{n_{-}}$

2) $\overline{\mathcal{N}_{\kappa, \zeta, c_{2}}(f, g)}=\int d z \mathcal{N}_{\kappa, \zeta, c_{2}}(f, g)=0$;

3) форма $\mathcal{N}_{\kappa, \zeta, c_{2}}(f, g)$ удовлетворяет тождеству Якоби.

ДокАЗАТЕЛЬство. Пункт 1 очевиден; пункт 2 верен, так как

$$
\int d z \mathcal{N}_{\kappa, \zeta, c_{2}}(f, g)=\bar{f} \bar{g} \int d z \mathcal{L}_{\kappa, \zeta, c_{2}}(z),
$$

а интеграл от $\mathcal{L}_{\kappa, \zeta, c_{2}}(z) \in \mathbf{D}_{n_{+}}^{n_{-}}$хорошо определен и равен нулю как интеграл четной функции по нечетному числу нечетных переменных.

Для доказательства пункта 3 заметим, что

$$
\begin{aligned}
\mathcal{N}_{\kappa, \zeta, c_{2}}\left(f, \mathcal{N}_{\kappa, \zeta, c_{2}}(g, h)\right)=\mathcal{M}_{\kappa}\left(f-\hbar^{2} \zeta_{C} \bar{f}, \mathcal{M}_{\kappa}\left(g-\hbar^{2} \zeta_{C} \bar{g}, h-\hbar^{2} \zeta_{C} \bar{h}\right)\right)+ \\
\quad+\mathcal{M}_{\kappa}\left(f-\hbar^{2} \zeta_{C} \bar{f}, c_{2} \bar{g} \bar{h}\right)+\left[\mathcal{M}_{\kappa}\left(f-\hbar^{2} \zeta_{C} \bar{f}, \zeta_{C}\right)+c_{2} \bar{f}\right] \overline{\mathcal{N}}_{\kappa, \zeta, c_{2}}(g, h)= \\
=\mathcal{M}_{\kappa}\left(f-\hbar^{2} \zeta_{C} \bar{f}, \mathcal{M}_{\kappa}\left(g-\hbar^{2} \zeta_{C} \bar{g}, h-\hbar^{2} \zeta_{C} \bar{h}\right)\right),
\end{aligned}
$$

и тождество Якоби следует из того, что форма $\mathcal{M}_{\kappa}(f, g)$ удовлетворяет тождеству Якоби для любых $f, g, h \in \mathbf{E}_{n_{+}}^{n_{-}}$.

Рассмотрим теперь форму

$$
\begin{aligned}
& \mathcal{N}_{\kappa_{[n]}, \zeta_{[n]}, c_{2[n]}}(f, g)=\mathcal{M}_{\kappa_{[n]}}\left(f-\hbar^{2} \zeta_{C[n]} \bar{f}, g-\hbar^{2} \zeta_{C[n]} \bar{g}\right)+c_{2[n]} \bar{f} \bar{g}= \\
& =\mathcal{M}_{\kappa_{[n]}}(f, g)-\hbar^{2} \mathcal{M}_{\kappa_{[n]}}\left(f, \zeta_{C[n]}\right) \bar{g}-\hbar^{2}(-1)^{\varepsilon(f) \varepsilon(g)} \mathcal{M}_{\kappa_{[n]}}\left(g, \zeta_{C[n]}\right) \bar{f}+ \\
& \quad+\mathcal{L}_{\kappa_{[n]}, \zeta_{[n]}, c_{2[n]}}(z) \bar{f} \bar{g}, \quad n \geqslant 1,
\end{aligned}
$$

где

$$
\begin{gathered}
\kappa_{[n]}=\sum_{k=1}^{n} \hbar^{2(k-1)} \kappa_{k}, \quad \zeta_{C[n]}(z)=\sum_{k=1}^{n} \hbar^{2(k-1)} \zeta_{C k}(z), \\
\varepsilon\left(\zeta_{C k}(z)\right)=1, \quad c_{2[n]}=\sum_{k=1}^{n} \hbar^{2 k} c_{2 k}, \quad c_{21}=0 .
\end{gathered}
$$

Мы полагаем, что

$$
\zeta_{C[n]} \in \mathbf{E}_{n_{+}}^{n_{-}},\left.\quad \mathcal{L}_{\kappa_{[n]}, \zeta_{[n]}, c_{2[n]}}\right|_{[n+1]}(z) \in \mathbf{D}_{n_{+}}^{n_{-}},
$$


где $\left.\left.A\left(\hbar^{2}\right)\right|_{[n]} \stackrel{\text { def }}{=} \sum_{k=0}^{n} A\left(\hbar^{2}\right)\right|_{k}$, а $\left.A\left(\hbar^{2}\right)\right|_{k}$ обозначает член порядка $\hbar^{2 k}$ в разложении функции $A\left(\hbar^{2}\right)$ в ряд Тейлора по $\hbar^{2}$. Тогда легко показать, что якобиатор $J\left(\mathcal{N}_{\kappa_{[n]}, \zeta_{[n]}, c_{2[n]}}, \mathcal{N}_{\kappa_{[n]}, \zeta_{[n]}, c_{2[n]}} \mid f, g, h\right)$ формы $\mathcal{N}_{\kappa_{[n]}, \zeta_{[n]}, c_{2[n]}}(f, g)$ обладает следующим свойством:

$$
\left.J\left(\mathcal{N}_{\kappa_{[n]}, \zeta_{[n]}, c_{2[n]}}, \mathcal{N}_{\kappa_{[n]}, \zeta_{[n]}, c_{2[n]}} \mid f, g, h\right)\right|_{[n+2]}=0
$$

(в этом якобиаторе члены порядка $\hbar^{2 k}, k \geqslant n+3$, могут быть плохо определены).

Выполняя преобразование эквивалентности $C(f, g) \rightarrow C_{T}(f, g)$ с $T(f)=f(z)-$ $\hbar^{2} t_{D 1}(f)+O\left(\hbar^{4}\right)$, перепишем $C(f, g)$ в виде

$$
C(f, g)=\mathcal{N}_{\kappa_{[1]}, \zeta_{[1]}, c_{2[1]}}(f, g)+\hbar^{4} C_{2}(f, g)-\left.\mathcal{L}_{\kappa_{[1]}, \zeta_{[1]}, c_{2[1]}}(z)\right|_{2} \bar{f} \bar{g}+O\left(\hbar^{6}\right) .
$$

Из условия $C(f, g) \in \mathbf{D}_{n_{+}}^{n_{-}}$следует, что $C_{2}(f, g) \in \mathbf{D}_{n_{+}}^{n_{-}}$.

Тождество Якоби (3) для $C(f, g, h)$ дает

$$
d_{2}^{\mathrm{ad}} C_{2}^{\prime}(f, g, h)=0, \quad C_{2}^{\prime}(f, g)=C_{2}(f, g)-\left.\hbar^{-4} \mathcal{L}_{\kappa_{[1]}, \zeta_{[1]}, c_{2[1]}}(z)\right|_{2} \bar{f} \bar{g}
$$

и, следовательно,

$$
C_{2}(f, g)=\left.\hbar^{-4} \mathcal{L}_{\kappa_{[1]}, \zeta_{[1]}, c_{2[1]}}(z)\right|_{2} \bar{f} \bar{g}+\frac{\kappa_{1} \kappa_{2}}{3} m_{2 \mid 1}(f, g)+c_{22} \bar{f} \bar{g}+d_{1}^{\mathrm{ad}} t_{2}(f, g) .
$$

Пусть

$$
z \cap \operatorname{supp}(f)=z \cap \operatorname{supp}(g)=\operatorname{supp}(f) \cap \operatorname{supp}(g)=\varnothing .
$$

Тогда мы имеем

$$
\widehat{C}_{2}(f, g)=\left[\left.\hbar^{-4} \mathcal{L}_{\kappa_{[1]}, \zeta_{[1]}, c_{2[1]}}(z)\right|_{2}+c_{22}\right] \bar{f} \bar{g} .
$$

Условие $\widehat{C}_{2}(f, g) \in \mathbf{D}_{n_{+}}^{n_{-}}$дает

$$
\left.\hbar^{-4} \mathcal{L}_{\kappa_{[1]}, \zeta_{[1]}, c_{2[1]}}(z)\right|_{2}+c_{22} \in \mathbf{D}_{n_{+}}^{n_{-}} .
$$

Таким образом, деформация возможна не для всех функций $\zeta_{C 1}(z)$, а только для тех, которые удовлетворяют условию (19).

Далее из условия $\left.d_{1}^{\text {ad }} t_{2}(f, g)\right] \in \mathbf{D}_{n_{+}}^{n_{-}}$мы находим (см. конец п. 4.3 работы [5]), что

$$
\begin{gathered}
t_{2}(f)=\zeta_{C 2}(z) \bar{f}+t_{D 2}(f), \\
\zeta_{C 2}(z) \in \mathbf{E}_{n_{+}}^{n_{-}} / \mathbf{Z}_{n_{+}}^{n_{-}}, \quad \varepsilon\left(\zeta_{C 2}\right)=1, \quad t_{D 2}(f) \in \mathbf{D}_{n_{+}}^{n_{-}} .
\end{gathered}
$$

Выполнив преобразование эквивалентности $C(f, g) \rightarrow C_{T}(f, g)$ с $T(f)=f(z)-$ $\hbar^{4} t_{D 2}(f)+O\left(\hbar^{6}\right)$, представим $C(f, g)$ в виде

$$
C(f, g)=\mathcal{N}_{\kappa_{[2]}, \zeta_{[2]}, c_{2[2]}}(f, g)+\hbar^{6} C_{3}(f, g)-\left.\mathcal{L}_{\kappa_{[2]}, \zeta_{[2]}, c_{2[2]}}(z)\right|_{3} \bar{f} \bar{g}+O\left(\hbar^{8}\right) .
$$

Из условия $C(f, g) \in \mathbf{D}_{n_{+}}^{n_{-}}$следует, что $C_{3}(f, g) \in \mathbf{D}_{n_{+}}^{n_{-}}$.

Тождество Якоби (3) для $C(f, g, h)$ дает

$$
d_{2}^{\mathrm{ad}} C_{3}^{\prime}(f, g, h)=0, \quad C_{3}^{\prime}(f, g)=C_{3}(f, g)-\left.\hbar^{-6} \mathcal{L}_{\kappa_{[2]}, \zeta_{[2]}, c_{2[2]}}(z)\right|_{3} \bar{f} \bar{g},
$$


откуда следует, что

$$
C_{3}(f, g)=\left.\hbar^{-6} \mathcal{L}_{\kappa_{[2]}, \zeta_{[2]}, c_{2[2]}}(z)\right|_{3} \bar{f} \bar{g}+\left(\frac{\kappa_{2}^{2}}{6}+\frac{\kappa_{1} \kappa_{3}}{3}\right) m_{2 \mid 1}(f, g)+c_{23} \bar{f} \bar{g}+d_{1}^{\text {ad }} t_{3}(f, g) .
$$

Пусть

$$
z \cap \operatorname{supp}(f)=z \cap \operatorname{supp}(g)=\operatorname{supp}(f) \cap \operatorname{supp}(g)=\varnothing .
$$

Тогда мы имеем

$$
\widehat{C}_{3}(f, g)=\left[\left.\hbar^{-6} \mathcal{L}_{\kappa_{[2]}, \zeta_{[2]}, c_{2[2]}}(z)\right|_{3}+c_{23}\right] \bar{f} \bar{g} .
$$

Условие $\widehat{C}_{3}(f, g) \in \mathbf{D}_{n_{+}}^{n_{-}}$дает

$$
\left[\left.\hbar^{-6} \mathcal{L}_{\kappa_{[2]}, \zeta_{[2]}, c_{2[2]}}(z)\right|_{3}+c_{22}\right] \in \mathbf{D}_{n_{+}}^{n_{-}} .
$$

Действуя аналогичным образом, мы окончательно находим, что с точностью до преобразований эквивалентности общий вид функционала $C(f, g)$, удовлетворяющего тождеству Якоби (3) (общий вид $*$-коммутатора), дается формулой

$$
\begin{gathered}
C(f, g)=\mathcal{N}_{\kappa_{[\infty]}, \zeta_{[\infty]}, c_{2[\infty]}(f, g),} \\
\zeta_{C \infty}(z) \in \mathbf{E}_{n_{+}}^{n_{-}} / \mathbf{Z}_{n_{+}}^{n_{-}}, \quad \varepsilon\left(\zeta_{C \infty}\right)=1, \quad\left[\mathcal{M}_{\kappa_{[\infty]}}\left(\zeta_{C[\infty]}, \zeta_{C[\infty]}\right)+c_{2[\infty]}\right] \in \mathbf{D}_{n_{+}}^{n_{-}} .
\end{gathered}
$$

ПРИЛОЖЕНИЕ

Здесь мы приведем точную формулировку и доказательство утверждения, о котором говорилось в сноске 2. Доказательство будет охватывать как случай супералгебры, генерируемой суперскобкой Пуассона, так и случай супералгебры, генерируемой антискобкой.

Введем некоторые обозначения. Постоянная симплектическая метрика $\omega_{\lambda}^{A B}$ несет здесь дополнительный индекс $\lambda$, равный 0 или 1 для случаев скобки и антискобки Пуассона, соответственно, невырожденна ${ }^{4)}$ и обладает свойствами

$$
\omega_{\lambda}^{B A}=-(-1)^{\left(\varepsilon_{A}+\lambda\right)\left(\varepsilon_{B}+\lambda\right)} \omega^{A B}, \quad \varepsilon\left(\omega_{\lambda}^{A B}\right)=\varepsilon_{A}+\varepsilon_{B}+\lambda .
$$

С помощью метрики $\omega_{\lambda}^{A B}$ обычным образом определяется скобка

$$
\begin{gathered}
{[f, g](z)=f(z) \overleftarrow{\partial_{A}} \omega_{\lambda}^{A B} \partial_{B} g(z),} \\
{[g, f]=-(-1)^{(\varepsilon(f)+\lambda)(\varepsilon(g)+\lambda)}[f, g], \quad \varepsilon([f, g])=\varepsilon(f)+\varepsilon(g)+\lambda,} \\
\int d z[f, g] h=(-1)^{\lambda+\lambda \varepsilon(g)} \int d z f[g, h]-2 \int d z f \Delta g h,
\end{gathered}
$$

где $\Delta=0$ при $\lambda=0$ и $\Delta=(1 / 2)(-1)^{\varepsilon_{A}} \omega_{1}^{A B} \partial_{A} \partial_{B}=\partial_{x^{i}} \partial_{\xi^{i}}$ при $\lambda=1$.

Лемма П.1. Пусть $\mathbf{m}: \mathbf{D}_{n_{+}}^{n_{-}} \rightarrow \mathbf{E}_{n_{+}}^{n_{-}}-$непрерывное линейное отображение. Для $g \in \mathbf{D}_{n_{+}}^{n_{-}}$определим функцию $\widetilde{\mathbf{m}}(z \mid g)$ на $\mathbb{R}^{n_{+}}$со значениями в $\mathbb{G}^{n_{-}}$соотношением $\widetilde{\mathbf{m}}(z \mid g)=\mathbf{m}(z \mid g(\cdot-z))$. Тогда $\widetilde{\mathbf{m}}$ является непрерывным линейным отображением из $\mathbf{D}_{n_{+}}^{n_{-}}$в $\mathbf{E}_{n_{+}}^{n_{-}}$.

4) При $\lambda=1$ требование невырожденности симплектической метрики $\omega_{1}^{A B}$ приводит к условию $n_{+}=n_{-}$. 
ЛЕмма П.2. Пусть обобщенная функция $m \in \mathbf{D}_{n_{1}^{+}+n_{2}^{+}}^{n_{-}^{-}+n_{-}^{-}}$является гладкой по первому аргументу, т.е. существует непрерывное линейное отображение $\mathbf{m}$ : $\mathbf{D}_{n_{2}^{+}}^{n_{2}^{-}} \rightarrow \mathbf{E}_{n_{1}^{+}}^{n_{1}^{-}}$maкoe, чmo

$$
\begin{gathered}
m\left(f \otimes^{\prime} g\right) \equiv \int d z d u m(z, u) g(u) f(z)=\int^{\mathrm{L}} d z \mathbf{m}(z \mid g) f(z), \\
\left(f \otimes^{\prime} g\right)(z, u)=g(u) f(z),
\end{gathered}
$$

для любых $f \in \mathbf{D}_{n_{1}^{+}}^{n_{1}^{-}}, g \in \mathbf{D}_{n_{2}^{+}}^{n_{-}^{-}}$. Здесь $\int^{\mathrm{L}}$ означает обычный интеграл Лебега при интегрировании по четным переменным. Тогда для любой $\varphi \in \mathbf{D}_{n_{1}^{+}+n_{2}^{+}}^{n_{1}^{-}+n_{-}^{-}}$имеем

$$
m(\varphi)=\int^{\mathrm{L}} d z \mathbf{m}(z \mid \varphi(\cdot, z))
$$

ЛЕмма П.3. Пусть непрерывные линейные отображсения $\mathbf{m}_{A}: \mathbf{D}_{n_{2}^{+}}^{n_{2}^{-}} \rightarrow \mathbf{E}_{n_{1}^{+}}^{n_{-}^{-}} m a-$ ковы, что $\mathbf{m}_{A}(z \mid g) \overleftarrow{\partial}_{B}=(-1)^{\varepsilon_{A} \varepsilon_{B}} \mathbf{m}_{B}(z \mid g) \overleftarrow{\partial}_{A}, 1 \leqslant A, B \leqslant n_{1}^{+}+n_{1}^{-}$, для любого $g \in \mathbf{D}_{n_{2}^{+}}^{n_{-}^{-}}$. Тогда существует такое непрерьвное линейное отображение $\mathbf{m}: \mathbf{D}_{n_{2}^{+}}^{n_{2}^{-}} \rightarrow$ $\mathbf{E}_{n_{1}^{+}}^{n_{-}^{-}}$, что $\mathbf{m}(z \mid g) \overleftarrow{\partial}_{A}=\mathbf{m}_{A}(z \mid g)$ для любого $g \in \mathbf{D}_{n_{2}^{+}}^{n_{-}^{-}}$

ДокАзАТЕЛЬСтво. Отображение $\mathbf{m}$, определенное формулой

$$
\mathbf{m}(z \mid g)=\int_{0}^{1} \mathbf{m}_{A}(t z \mid g) z^{A} d t
$$

удовлетворяет всем условиям леммы.

Дифференциал

$d_{1}^{\text {ad }} M_{1}(z \mid f, g)=\left[M_{1}(z \mid f), g(z)\right]-(-1)^{(\varepsilon(f)+\lambda)(\varepsilon(g)+\lambda)}\left[M_{1}(z \mid g), f(z)\right]-M_{1}(z \mid[f, g])$

будем рассматривать как оператор из $\mathbf{D}_{2 n^{+}}^{\prime 2 n^{-}}$в $\mathbf{D}_{3 n^{+}}^{\prime 3 n^{-}}$. Пусть $M_{1}(z \mid u)$ - такая обобщенная функция, что $d_{1}^{\text {ad }} M_{1}$ является гладкой по первому аргументу, т.е. существует такое непрерывное линейное отображение $\mathbf{M}_{2}: \mathbf{D}_{n_{+}}^{n_{-}} \rightarrow \mathbf{E}_{n_{+}}^{n_{-}}$, что

$$
\left(d_{1}^{\mathrm{ad}} M_{1}\right)\left(h \otimes^{\prime} \psi\right)=\int d z d_{1}^{\mathrm{ad}} M_{1}(z \mid \psi) h(z)=\int^{\mathrm{L}} d z \mathbf{M}_{2}(z \mid \psi) h(z)
$$

для любых $h \in \mathbf{D}_{n_{+}}^{n_{-}}, \psi \in \mathbf{D}_{2 n^{+}}^{2 n^{-}}$.

Обозначим через $S_{R}$ и $\bar{S}_{R}$ соответственно открытый и замкнутый шары радиуса $R$ с центром в начале координат. Обозначим через $\mathbf{D}\left(\bar{S}_{R}\right)$ подпространство $\mathbf{D}_{n_{+}}^{n_{-}}$, состоящее из таких $f$, что $\operatorname{supp} f \subset \bar{S}_{R}$.

Лемма П.4. Пусть $R>0, g, h \in \mathbf{D}\left(\bar{S}_{R}\right), \partial_{A} g(0)=\partial_{A} \partial_{B} g(0)=0$ для всех $A, B ; \quad f_{A}^{R} \in \mathbf{D}_{n_{+}}^{n_{-}}$такова, что $f_{A}^{R}(z)=-\omega_{\lambda, A B} z^{B} \quad\left[\varepsilon\left(f_{A}^{R}\right)=\varepsilon_{A}+\lambda\right]$ в некоторой окрестности $\bar{S}_{2 R}, \omega_{\lambda, A B} \omega_{\lambda}^{B C}=\delta_{A}^{C}$. Тогда

$$
\int^{\mathrm{L}} d z \mathbf{M}_{2}\left(z \mid f_{A}^{R} \otimes^{\prime} g(\cdot-z)\right) h(z)=\int d z d u M_{1}(z \mid u) g(u-z) \partial_{A} h(z) .
$$


ДокАЗАТЕЛЬСтво. В силу леммы П.2 имеем

$$
\int^{\mathrm{L}} d z \mathbf{M}_{2}\left(z \mid f_{A}^{R} \otimes^{\prime} g(\cdot-z)\right) h(z)=(-1)^{\varepsilon\left(f_{A}^{R}\right) \varepsilon(g)}\left(d_{1}^{\mathrm{ad}} M_{1}\right)\left(\varphi_{A}\right),
$$

где $\varphi_{A}(v, w, z)=f_{A}^{R}(v) g(w-z) h(z)$. Легко видеть, что

$$
\left(d_{1}^{\mathrm{ad}} M_{1}\right)(\varphi)=\int d z d u M_{1}(z \mid u) L \varphi(u, z),
$$

где оператор $L: \mathbf{D}_{3 n^{+}}^{3 n^{-}} \rightarrow \mathbf{D}_{2 n^{+}}^{2 n^{-}}$действует на $\varphi(v, w, z)$ по правилу

$$
\begin{gathered}
L \varphi(u, z)=\varphi(v, w, z)\left[\left.\overleftarrow{l}_{w z}\right|_{v=u, w=z}-\left.(-1)^{\lambda} \overleftarrow{l}_{v z}\right|_{v=z, w=u}-\left.(-1)^{\lambda} \overleftarrow{l}_{v, w}\right|_{v=w=u}+\right. \\
\left.+\left.2 \overleftarrow{\Delta}^{w}\right|_{v=u, w=z}+\left.2 \overleftarrow{\Delta}^{v}\right|_{v=z, w=u}\right](-1)^{\lambda(\varepsilon(g)+\varepsilon(h))} \\
\overleftarrow{l}_{v z}=\overleftarrow{\partial}_{A}^{v} \omega_{\lambda}^{A B}(-1)^{\varepsilon_{B}} \overleftarrow{\partial}_{B}^{z}
\end{gathered}
$$

Применяя $L$ к функции $\varphi_{A}$ указанного выше вида и пользуясь условием $\partial g(0)=$ $\partial^{2} g(0)=0$, получаем

$$
\begin{aligned}
\left(L \varphi_{A}\right)(u, z)=- & (-1)^{\left(\lambda+\varepsilon_{A}\right) \varepsilon(g)} f_{A}^{R}(z) \overleftarrow{\partial}_{B} \omega_{\lambda}^{B C} g(u-z) \partial_{C} h(z)+ \\
& +\omega_{\lambda}^{B C}\left[f_{A}^{R}(z) \overleftarrow{\partial}_{B}-f_{A}^{R}(u) \overleftarrow{\partial}_{B}\right] \partial_{C}^{u} g(u-z) h(z)+2 f_{A}^{R}(z) \overleftarrow{\Delta} g(u-z) h(z)
\end{aligned}
$$

Из условия для носителей $h$ и $g$ следует, что второй и третий члены отличны от нуля лишь при $|x| \leqslant R$ и $|y| \leqslant 2 R$. При этих условиях второй и третий члены тождественно равны нулю и, кроме того, $\varepsilon\left(f_{A}^{R}\right)=\varepsilon_{A}+\lambda$. Вычисление первого члена дает

$$
\left(L \varphi_{A}\right)(u, z)=(-1)^{\left(\lambda+\varepsilon_{A}\right) \varepsilon(g)} g(u-z) \partial_{A} h(z) .
$$

Подстановка полученного соотношения в (П.1) приводит к требуемому равенству.

Пусть $\psi(z), \varepsilon(\psi)=0$ - гладкая функция с компактным носителем на $\mathbb{R}^{n}$, равная единице в некоторой окрестности начала координат и нулю при $|x| \geqslant 1$. Для $g \in \mathbf{D}_{n_{+}}^{n_{-}}$ положим

$$
\hat{g}(z)=g(z)-\psi(z)\left[z^{A} \partial_{A}+\frac{1}{2} z^{A} z^{B} \partial_{B} \partial_{A}\right] g(0) .
$$

Очевидно, что $\partial \hat{g}(0)=\partial^{2} \hat{g}(0)$.

ЛЕмма П.5. Существует такое непрерывное линейное отображение $\mathbf{m}_{1 A}$ : $\mathbf{D}_{n_{+}}^{n_{-}} \rightarrow \mathbf{E}_{n_{+}}^{n_{-}}, \quad f \in \mathbf{D}_{n_{+}}^{n_{-}}, \mathbf{m}_{1 A}(z \mid f) \in \mathbf{E}_{n_{+}}^{n_{-}}$, чmo

$$
\int d z d u M_{1}(z \mid u) \hat{g}(u-z) \partial_{A} h(z)=\int^{\mathrm{L}} d z \mathbf{m}_{1 A}(z \mid g) h(z)
$$

для любъх $g, h \in \mathbf{D}_{n_{+}}^{n_{-}}$.

ДокАЗАтЕльство. Для $R>0$ определим линейное отображение $\mathbf{M}_{1 A}^{R}: \mathbf{D}_{n_{+}}^{n_{-}} \rightarrow$ $\mathbf{E}_{n_{+}}^{n_{-}}$соотношением

$$
\mathbf{M}_{1 A}^{R}(z \mid g)=\mathbf{M}_{2}\left(z \mid f_{A}^{R} \otimes g(\cdot-z)\right), \quad g \in \mathbf{D}_{n_{+}}^{n_{-}},
$$


где $f_{A}^{R}$ - функция со свойствами, указанными в условии леммы П.4. Из леммы П.1 следует, что отображение $\mathbf{M}_{1 A}^{R}$ непрерывно. Обозначим через $P^{R}$ линейный оператор из $\mathbf{D}\left(\bar{S}_{R}\right)$ в $\mathbf{D}_{n_{+}}^{n_{-}}$, переводящий $g$ в $\hat{g}$. Пусть $Q^{R}: \mathbf{E}_{n_{+}}^{n_{-}} \rightarrow \mathbf{E}\left(S_{R}\right)$ - линейный оператор, ставящий в соответствие гладкой функции на $\mathbb{R}^{n}$ ее сужение на $S_{R}$. Определим линейное отображение $\mathbf{m}_{1 A}^{R}: \mathbf{D}\left(\bar{S}_{R}\right) \rightarrow \mathbf{E}\left(S_{R}\right)$ как композицию: $\mathbf{m}_{1 A}^{R}=Q^{R} \circ \mathbf{M}_{1 A}^{R} \circ P^{R}$. Таким образом, $\mathbf{m}_{1 A}^{R}$ переводит $g \in \mathbf{D}\left(\bar{S}_{R}\right)$ в сужение функции $z \rightarrow \mathbf{M}_{2}\left(z \mid f_{A}^{R} \otimes^{\prime} \hat{g}(\cdot-z)\right)$ на шар $S_{R}$. Так как все операторы $P^{R}, Q^{R}$ и $\mathbf{M}_{1 A}^{R}$ непрерывны, то отображение $\mathbf{m}_{1 A}^{R}$ непрерывно. Если $R>1, g \in \mathbf{D}\left(\bar{S}_{R}\right)$ и $\operatorname{supp} h \subset S_{R}$, то из леммы П.4 вытекает, что

$$
\int_{S_{R}}^{\mathrm{L}} d z \mathbf{m}_{1 A}^{R}(z \mid g) h(z)=\int d z d u M_{1}(z \mid u) \hat{g}(u-z) \partial_{A} h(z) .
$$

Из этого равенства следует, что если $R^{\prime} \geqslant R>1$ и $g \in \mathbf{D}\left(\bar{S}_{R}\right)$, то $\mathbf{m}_{1 A}^{R}(z \mid g)$ является сужением $\mathbf{m}_{1 A}^{R^{\prime}}(z \mid g)$ на $S_{R}$. Таким образом, отображения $\mathbf{m}_{1 A}^{R}$ при разных $R$ согласованны между собой и, следовательно, определяют искомое отображение $\mathbf{m}_{1 A}: \mathbf{D}_{n_{+}}^{n_{-}} \rightarrow \mathbf{E}_{n_{+}}^{n_{-}}$.

Из формулы (П.2) следует

$$
\begin{aligned}
\int^{\mathrm{L}} d z \partial_{A} \mathbf{m}_{1 B}(z \mid g) h(z) & =-(-1)^{\varepsilon_{B}} \int d z d u M_{1}(z \mid u) \hat{g}(u-z) \partial_{A} \partial_{B} h(z)= \\
& =(-1)^{\varepsilon_{A} \varepsilon_{B}+\varepsilon_{A}+\varepsilon_{B}} \int^{\mathrm{L}} d z \partial_{B} \mathbf{m}_{1 A}(z \mid g) h(z) .
\end{aligned}
$$

Это означает, что $\partial_{A}\left[(-1)^{\varepsilon_{B}} \mathbf{m}_{1 B}(z \mid g)\right]$ и $(-1)^{\varepsilon_{A} \varepsilon_{B}} \partial_{B}\left[(-1)^{\varepsilon_{A}} \mathbf{m}_{1 A}(z \mid g)\right]$ совпадают как обобщенные функции, а следовательно, совпадают и поточечно. Поэтому в силу леммы П.3 существует такое непрерывное линейное отображение $\mathbf{m}_{1}: \mathbf{D}_{n_{+}}^{n_{-}} \rightarrow \mathbf{E}_{n_{+}}^{n_{-}}$, что $(-1)^{\varepsilon_{A}} \mathbf{m}_{1}(z \mid g) \overleftarrow{\partial}_{A}=\mathbf{m}_{1 A}(z \mid g)$ для любого $g \in \mathbf{D}_{n_{+}}^{n_{-}}$. Положим $\widetilde{M}(z, u)=M_{1}(z \mid$ $u+z)$. В силу (П.2) имеем

$$
\begin{array}{r}
\int^{\mathrm{L}} d z(-1)^{\varepsilon_{A}} \mathbf{m}_{1}(z \mid g) \overleftarrow{\partial}_{A} h(z)=\widetilde{M}\left(\partial_{A} h \otimes^{\prime} \hat{g}\right)=\widetilde{M}\left(\partial_{A} h \otimes^{\prime} g\right)- \\
-(-1)^{\varepsilon(g)\left[\varepsilon_{A}+\varepsilon(h)\right]}\left[\mu^{B}\left(\partial_{A} h\right) \partial_{B} g(0)+\frac{1}{2} \mu^{B C}\left(\partial_{A} h\right) \partial_{C} \partial_{B} g(0)\right]
\end{array}
$$

где обобщенные функции $\mu^{B}, \mu^{B C} \in \mathbf{D}_{n_{+}}^{n^{-}}$определяются соотношениями

$$
\mu^{B}(h)=\int d z d u \widetilde{M}(z, u) h(z) \psi(u) u^{B}, \quad \mu^{B C}(h)=\int d z d u \widetilde{M}(z, u) h(z) \psi(u) u^{B} u^{C}
$$

Пусть $\tilde{m} \in \mathbf{D}_{2 n^{+}}^{\prime 2 n^{-}}$- обобщенная функция двух переменных, соответствующая отображению $\mathbf{m}_{1}$, т.е. обобщенная функция, определяемая соотношением $\widetilde{m}\left(h \otimes^{\prime} g\right)=$ $\int^{\mathrm{L}} d z \mathbf{m}_{1}(z \mid g) h(z)$. В силу (П.3) имеем

$$
\partial_{A}^{z} \widetilde{m}(z, u)=-\partial_{A}^{z} \widetilde{M}(z, u)+\partial_{A}^{z} \mu^{B}(z) \partial_{B} \delta(u)+\partial_{A}^{z} \mu^{B C}(z) \partial_{C} \partial_{B} \delta(u) .
$$


Следовательно, существует такая обобщенная функция $a \in \mathbf{D}_{n_{+}}^{n^{-}}$, что

$$
\widetilde{M}(z, u)=-\widetilde{m}(z, u)+\mu^{B}(z) \partial_{B} \delta(u)+\mu^{B C}(z) \partial_{C} \partial_{B} \delta(u)+a(u) .
$$

Пусть $m_{1}(z \mid u)=\widetilde{m}(z, u-z)$. Возвращаясь к исходным переменным, находим

$$
M_{1}(z \mid u)=-m_{1}(z \mid u)+\mu^{B}(z) \partial_{B} \delta(u-z)+\mu^{B C}(z) \partial_{C} \partial_{B} \delta(u-z)+a(u-x) .
$$

В силу леммы П.2

$$
\begin{aligned}
m_{1}\left(h \otimes^{\prime} g\right) & =\int d z d u \widetilde{m}(z, u) g(u) h(z)=\int d z d u \widetilde{m}(z, u) g(u+z) h(z)= \\
& =\int d z \mathbf{m}_{1}(z \mid g(\cdot+z)) h(z), \quad g, h \in \mathbf{D}_{n_{+}}^{n_{-}} .
\end{aligned}
$$

Для $g \in \mathbf{D}_{n_{+}}^{n_{-}}$определим функцию $\widetilde{\mathbf{m}}(g)$ на $\mathbb{R}^{n}$ соотношением

$$
\widetilde{\mathbf{m}}(g)(z)=\mathbf{m}_{1}(z \mid g(\cdot+z)) .
$$

В силу леммы П.1 (с измененным знаком в формулировке) $\widetilde{\mathbf{m}}$ является непрерывным отображением из $\mathbf{D}_{n_{+}}^{n_{-}}$в $\mathbf{E}_{n_{+}}^{n_{-}}$, а из (П.4) следует $m_{1}\left(h \otimes^{\prime} g\right)=\int d z \widetilde{\mathbf{m}}(g)(z) h(z)$. Это означает, что $m_{1}(z, u)$ является гладкой по первому аргументу. Так как свертка обобщенной функции с пробной функцией является гладкой функцией, то обобщенная функция $a(u-z)$ является гладкой по $z$. Таким образом, мы приходим к следующему утверждению.

ЛЕмма П.6. Пусть $M_{1}(z \mid u)$ - такая обобщенная функиия, что $d_{1}^{\mathrm{ad}} M_{1}$ является гладкой по первому аргументу. Тогда $M_{1}$ представима в виде

$$
\begin{gathered}
M_{1}(z \mid u)=M_{1}^{\prime}(z \mid u)+M_{1 \text { loc }}(z \mid u), \\
M_{1 \text { loc }}(z \mid u)=\mu^{B}(z) \partial_{B} \delta(u-z)+\mu^{B C}(z) \partial_{C} \partial_{B} \delta(u-z),
\end{gathered}
$$

где $\mu^{B}, \mu^{B, C} \in \mathbf{D}_{n_{+}}^{n^{-}}$, a $M^{\prime} \in \mathbf{D}_{2 n^{+}}^{\prime 2 n^{-}}$является гладкой по первому аргументу.

Рассмотрим соотношение

$$
M_{2}(z \mid f, g)-d_{1}^{\text {ad }} M_{1}^{\prime}(z \mid f, g)=d_{1}^{\text {ad }} M_{1 \text { loc }}(z \mid f, g) .
$$

Так как левая сторона этого соотношения является непрерывным отображением из $\mathbf{D}_{2 n^{+}}^{2 n^{-}}$в $\mathbf{E}_{n_{+}}^{n_{-}}$, форма $d_{1}^{\text {ad }} M_{1 \text { loc }}(z \mid f, g)$ также является непрерывным отображением из $\mathbf{D}_{2 n^{+}}^{2 n^{-}}$в $\mathbf{E}_{n_{+}}^{n_{-}}$. Записывая явное выражение для $d_{1}^{\text {ad }} M_{1 \text { loc }}(z \mid f, g)$ и рассматривая коэффициенты перед $\partial^{k} f \partial^{p} g$ (или, эквивалентно, выбирая $f$ и $g$ пропорциональными различным степеням переменной $z)$, мы находим, что $\mu^{B}, \mu^{B C} \in \mathbf{E}_{n_{+}}^{n_{-}}$.

Таким образом, нами доказана следующая теорема.

ТЕОРема П.1. Пусть форма $M_{2}(z \mid f, g)$ - разделъно-непрерывное отображение из $\mathbf{D}_{n^{+}}^{n^{-}}$в $\mathbf{E}_{n_{+}}^{n_{-}}$(является гладкой по $z$ ) и имеет место равенство

$$
M_{2}(z \mid f, g)=d_{1}^{\mathrm{ad}} M_{1}(z \mid f, g) .
$$

Тогда форма $M_{1}(z \mid f)$ - непрерывное отображение из $\mathbf{D}_{n_{+}}^{n_{-}}$в $\mathbf{E}_{n_{+}}^{n_{-}}$(является гладкой no $z)$. 
Благодарности. Настоящая работа поддержана РФФИ (гранты № 02-02-16944 (И. Тютин), № 02-02-17067 (С. Конштейн) и № 02-02-16946 (А. Смирнов)) и INTAS (гранты № 03-51-6346 (А. Смирнов) и № 00-00-262 (И. Тютин)), а также грантом LSS-1578.2003.2.

\section{Список литературы}

[1] F. Bayen, M. Flato, C. Fronsdal, A. Lichnerovich, D. Sternheimer, Ann. Phys., 111 (1978), 61; 111; М.В. Карасев, В.П. Маслов, Нелинейные скобки Пуассона. Геометрия и квантование, Наука, M., 1991; B. Fedosov, Deformation Quantization and Index Theory, Akademie, Berlin, 1996; M. Kontsevich, Lett. Math. Phys., 66:3 (2003), 157; q-alg/9709040.

[2] Д. А. Лейтес, И. М. Щепочкина, ТМФ, 126:3 (2001), 339.

[3] И. В. Тютин, ТМФ, 128:3 (2001), 515.

[4] I. A. Batalin, I. V. Tyutin, General local solution to the Jacoby cyclic equation (unpublished).

[5] С. Е. Конштейн, А. Г. Смирнов, И. В. Тютин, ТМФ, 143:2 (2005), 163; hep-th/0312109.

[6] M. Gerstenhaber, Ann. Math., 79 (1964), 59; 99 (1974), 257.

Поступила в редакцию 12.12 .2005 